\title{
Effects of Dexmedetomidine as an Analgesic Adjuvant for Surgery of Femur Fracture: A Systematic Review and Meta-Analysis
}

\author{
Shan Deng ${ }^{a}$ Yonghao Yu ${ }^{b}$ \\ ${ }^{a}$ Anesthesiology Department, Tianjin Hospital, Tianjin, China; ${ }^{b}$ Anesthesiology Department, Tianjin Medical \\ University General Hospital, Tianjin, China
}

\section{Keywords}

Dexmedetomidine $\cdot$ Surgery $\cdot$ Femur fracture $\cdot$ Meta-

analysis

\begin{abstract}
Patients who undergo surgery of femur fracture suffer the excruciating pain. Dexmedetomidine (DEX) is a unique a2adrenergic receptor agonist with sedative and analgesic properties, whose efficacy and safety are still unclear for surgery of femur fracture. Randomized controlled trials comparing the effects of addition of DEX to general or local anesthesia in surgery of femur fracture were searched from MEDLINE, EMBASE, and the Cochrane Library database. Patients who received DEX infusion had a significant longer time to rescue analgesia compared with those without DEX coadministration. DEX treatment seemed to reduce the visual analog score; however, the significance did not reach any statistical difference. DEX as an analgesic adjuvant did not reduce the onset of sensory block time, shorten the time to achieve maximum sensory block level, and provide a longer duration of sensory block. The difference in mean sedation scores between 2 groups was not statistically significant. As for adverse effects, DEX therapy significantly in-
\end{abstract}

creased the rate of hypotension. In conclusion, dexmedetomidine as a local anesthetic adjuvant in femur fracture surgery had a longer duration of rescue analgesia. However, the incidence of hypotension was markedly increased in these patients. It was worth noting that the evidence was of low to moderate quality.

(C) 2021 S. Karger AG, Basel

\section{Introduction}

Patients who undergo surgery of femur fracture always suffer the excruciating pain, which can affect psychological state and delay the time of discharge [1]. Over the years, the invention for pain that relates to general and local anesthesia includes lidocaine, bupivacaine, opioids, and nonsteroidal anti-inflammatory drugs [2-4]. However, these anesthetics may result in a high risk of respiratory depression and increase the risk of opioid-induced hyperalgesia in patients with surgery of femur fracture [2]. In order to maximize pain relief and decrease opioid requirement, multimodal applications using different classes of analgesic adjuvants are currently recommended. karger@karger.com www.karger.com/pha

(C) 2021 S. Karger AG, Base

Karger"
Correspondence to:

Yonghao Yu, yonghaoyutj@163.com 


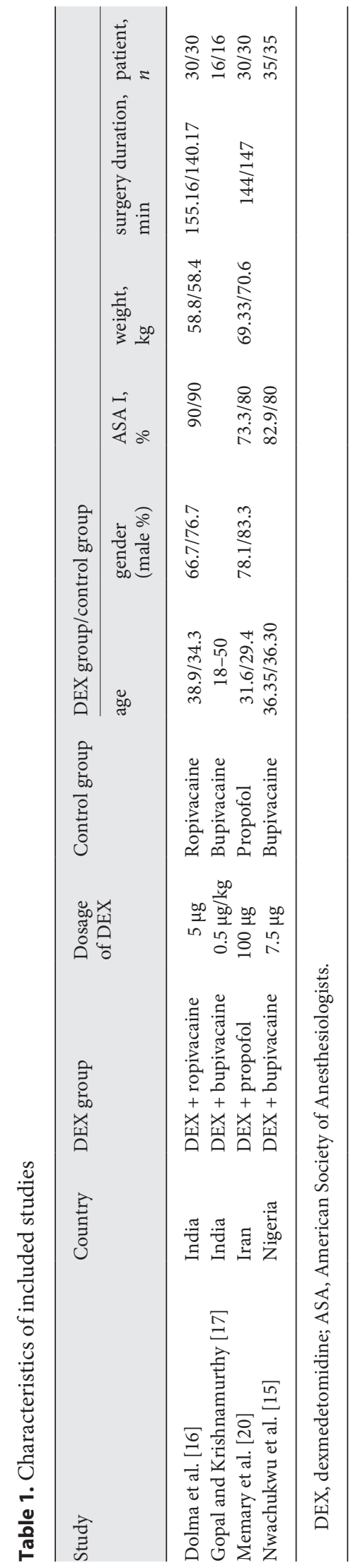

Dexmedetomidine (DEX) is a unique $\alpha 2$-adrenergic receptor agonist with sedative and analgesic properties [5]. DEX exerts its sedative effect by activation of central pre- and postsynaptic $\alpha 2$-receptors in the locus coeruleus, inducing a state mimicking natural sleep. DEX is rapidly distributed all over the body and is eliminated mainly by the liver $[6,7]$. On the contrary, it may increase the risk of hemodynamic alterations, which appear to be dose dependent $[8,9]$. In recent years, DEX as an adjuvant to anesthetics has been added with local anesthetic agents, propofol or opioids, to augment their efficacy [10-14]. A reduction of doses of anesthetics is also expected to reduce adverse events. At present, a number of studies were conducted in patients with fracture of femur surgery to evaluate the efficacy and side effects of DEX as an adjuvant [15-17]. Some provided evidence that treatment with DEX exhibited analgesic and sedative effects, while others did not find the beneficial effect of DEX. It is not clear exactly whether this agent provides additional benefit in patients with femoral fracture. In this study, we undertook a meta-analysis to summarize and evaluate the efficacy and safety of dexmedetomidine therapy in patients undergoing femoral fracture surgery for the first time.

\section{Methods}

Data Sources and Searches

We identified relevant studies from the following data sources: MEDLINE (from 1950 to August 2020), EMBASE (from 1970 to August 2020), the Cochrane Library database (to August 2020), and the ClinicalTrials.gov website (to August 2020) with the text words of "randomized controlled trial (RCT)," "dexmedetomidine," "femur," and "femoral." RCTs were conducted following the PRISMA guidelines (online suppl. Table 1; for all online suppl. material, see www.karger.com/doi/10.1159/000515788) and restricted to those published in English language. Relevant reference lists were also scanned from identified trials.

\section{Study Selection}

In this study, RCTs regarding comparison of effects of DEX in patients with surgery of femur fracture were collected. Inclusion criteria for studies were as follows: (1) the intervention was dexmedetomidine; (2) study outcomes included time to rescue analgesia, visual analog score (VAS) for pain assessment, sensory block onset time, time to achieve maximum sensory block, sensory block duration, sedation score, and/or side effects of dexmedetomidine; (3) patients undergone femoral fracture surgery; (4) study design was RCT. The studies with lack of available data were excluded.

Data Extraction and Risk of Bias

Two investigators (Shan Deng and Yonghao Yu) extracted information using a standard data extraction form independently. The information included baseline patient characteristics, intervention method, dose of drug, surgery duration, outcome events, 
Fig. 1. Process for identifying studies eligible for the meta-analysis.

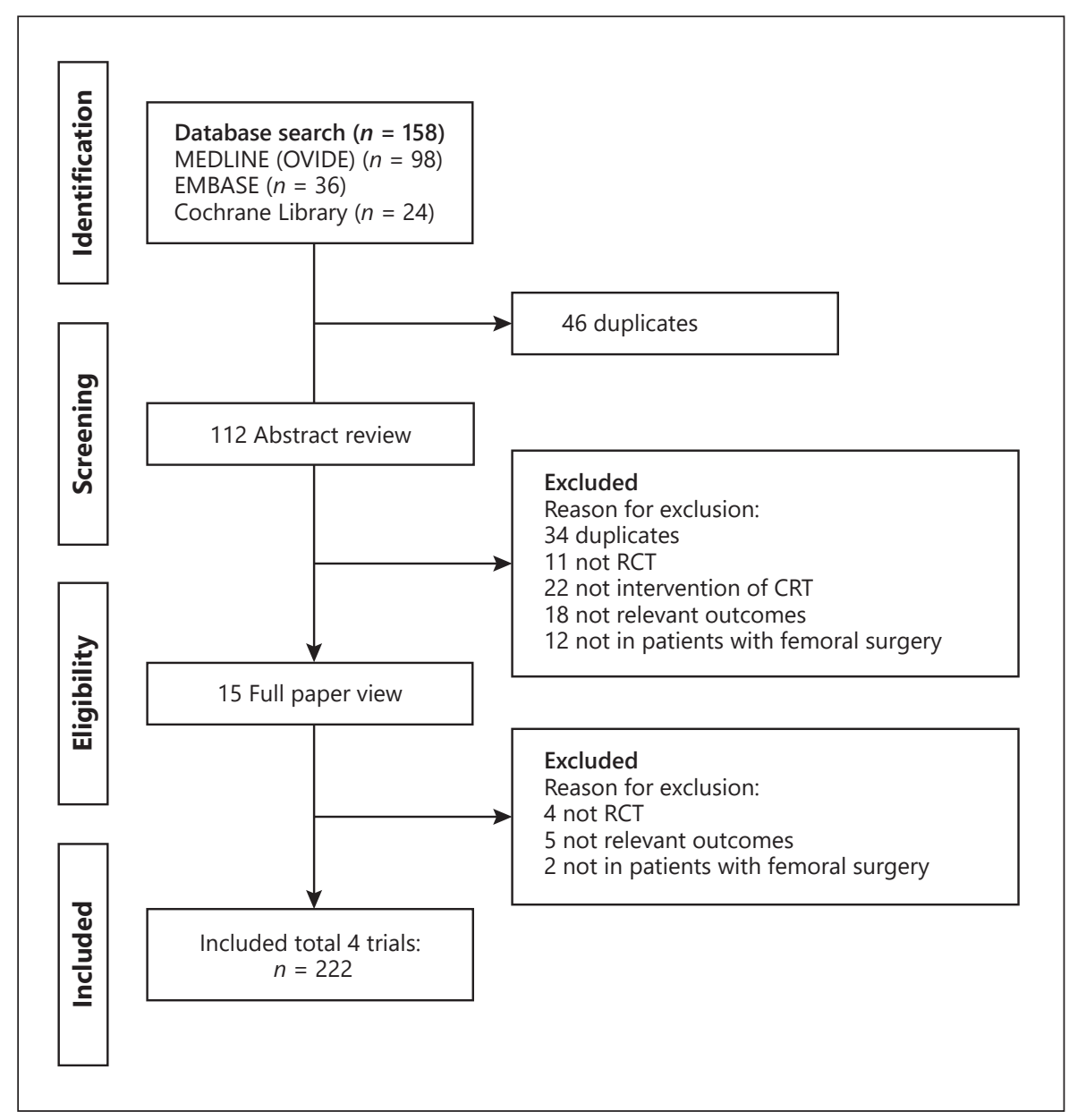

and adverse events. The risk of bias of included RCTs was judged according to the Cochrane Collaboration tool for assessing the risk of bias [18]. The Grading of Recommendations Assessment, Development and Evaluation (GRADE) approach was also used to assess of the quality of the evidence [19]. Any disagreements were resolved by discussion between 2 investigators.

Data Synthesis and Statistical Analyses

We calculated weighted mean difference and standard deviation between groups which were applied for continuous variables of time to rescue analgesia, VAS for pain assessment, sensory efficacy, and sedation score. The relative risk (RR) and 95\% confidence interval (CI) were applied to adverse effects of hypotension, bradycardia, and nausea and vomiting using a random-effect model. We analyzed heterogeneity by $I^{2}$ statistic to describe the percentage of variability. A value of $I^{2}>50 \%$ was considered a substantial level of heterogeneity. Once heterogeneity was noted, publication bias was assessed by the Begg Funnel plot. The results were considered significant with 2 -sided $p<0.05$. We performed this meta-analysis with the STATA, version 12.0, and Review Manager 5.1 .

Dexmedetomidine in Surgery of Femur Fracture

\section{Results}

\section{Study Characteristics}

A total of 158 articles relevant to dexmedetomidine administration in patients undergoing femoral surgery were retrieved. Eventually, as shown in Figure 1, 4 studies with 222 patients were included in this meta-analysis according to the above criteria [15-17, 20]. Baseline characteristics are shown in Table 1 . The mean ages ranged from 29 to 50 years, and the male percentage was $66-83 \%$. The majority of patients belonged to American Society of Anesthesiologists (ASA) Physical status Class I, with a high percentage of $73-90 \%$. The mean duration of surgery was $150 \mathrm{~min}$. The patients' number in each study ranged from 32 to 70 . Patients in the intervention group were treated with ropivacaine, bupivacaine, or propofol in combination with DEX, while the control group was treated without DEX. Dosage regimen varied among the included studies, with a dosage of $0.5 \mu \mathrm{g} / \mathrm{kg}$ in 1 study, $5-7$ $\mu \mathrm{g}$ in 2 studies, and $100 \mu \mathrm{g}$ in 1 study. 


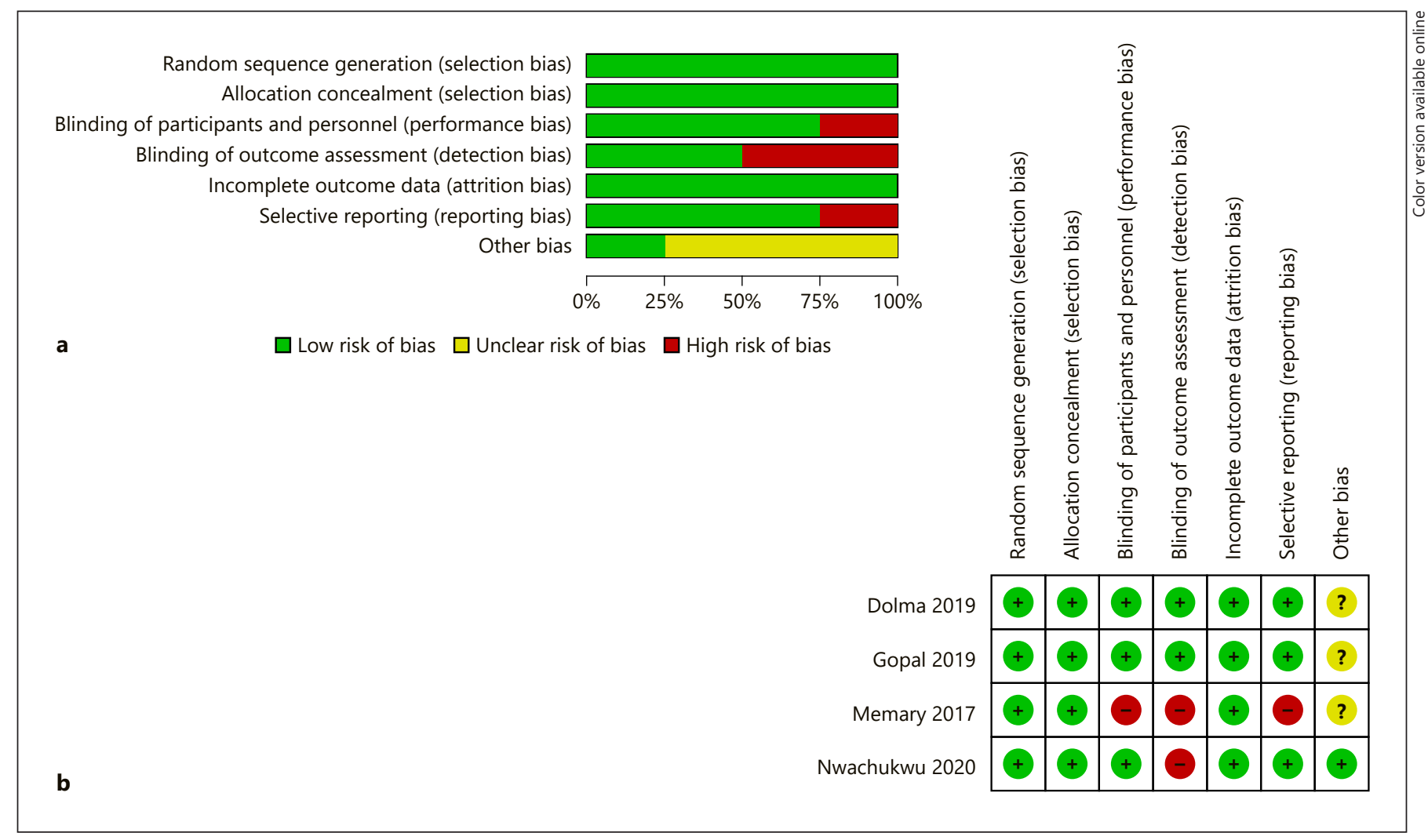

Fig. 2. Risk of bias graph (a) and risk of bias summary (b).

\section{Risk of Bias}

We evaluated the risk of bias of each study by using the Cochrane risk of bias tool, including sequence generation, allocation concealment, performance bias, detection bias, incomplete outcome data, selective reporting, and other possible sources of bias. The summary of the risk of bias is presented in Figure 2. GRADE summary of the quality of the evidence for each outcome is shown in Table 2 .

\section{Analgesic Efficacy}

In terms of analgesic efficacy, it was assessed by the time to rescue analgesia, VAS, time to sensory block onset, time to achieve maximum sensory block, and sensory block duration. Three RCTs reported the impact of DEX administration on the time to rescue analgesia. Patients who received DEX had a significant longer time to rescue analgesia (MD $202 \mathrm{~min}$; 95\% CI: 72.79-333.09 $\mathrm{min} ; p=$ $0.002 ; I^{2}=99 \%, p<0.001$; Fig. 3) compared with those who received control.
Pain degree was recorded by the VAS, which was assessed in 2 studies. DEX treatment seemed to reduce the VAS; however, the significance did not reach any statistical difference $(-2.44 ; 95 \% \mathrm{CI}:-6.03$ to $1.15 \mathrm{~min} ; p=0.18$; Fig. 4).

The sensory block efficacy was assessed by sensory block onset time, time to achieve maximum sensory block, or sensory block duration in 2 studies. Compared with the control, DEX therapy did not reduce the onset of sensory block time ( $0.37 \mathrm{~min}$; $95 \% \mathrm{CI}:-2.81$ to $3.55 \mathrm{~min}$; $p=0.82 ; I^{2}=78 \%, p=0.03$; online suppl. Fig. 1$)$, shorten the time to achieve maximum sensory block level $(2.22$ $\min ; 95 \% \mathrm{CI}:-1 / 25$ to $5.7 \mathrm{~min} ; p=0.21 ; I^{2}=47 \%, p=0.17$; online suppl. Fig. 2), and provide a longer duration of sensory block (210.59 $\mathrm{min}$; $95 \% \mathrm{CI}$ : -114.01 to 535.19 $\min ; p=0.20 ; I^{2}=99 \%, p<0.001$; online suppl. Fig. 3 ).

\section{Sedative Efficacy}

The Ramsay Sedation Score is often used for assessing sedative efficacy. The Ramsay Sedation Score was assessed by 2 studies. The difference in mean sedation 


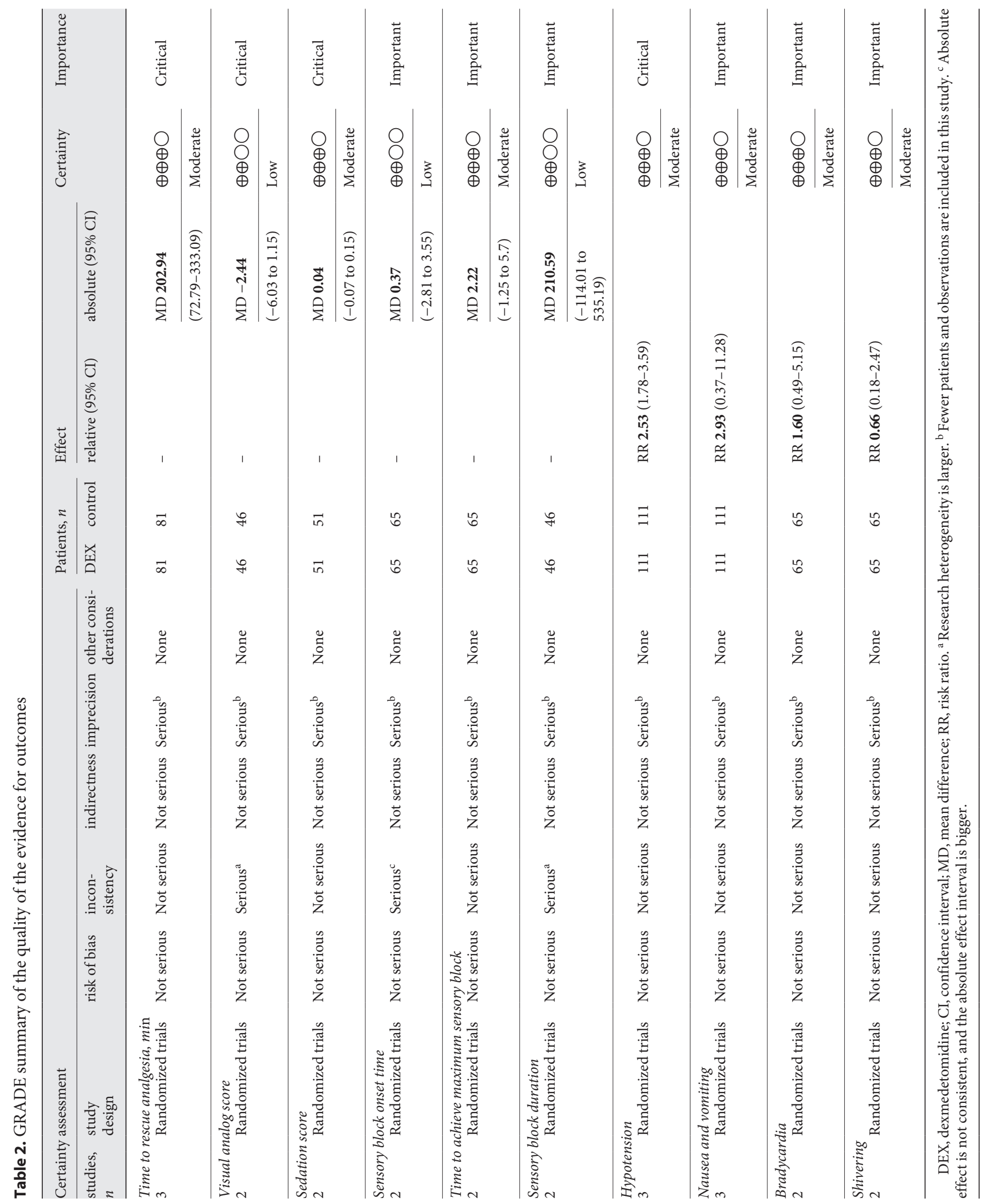




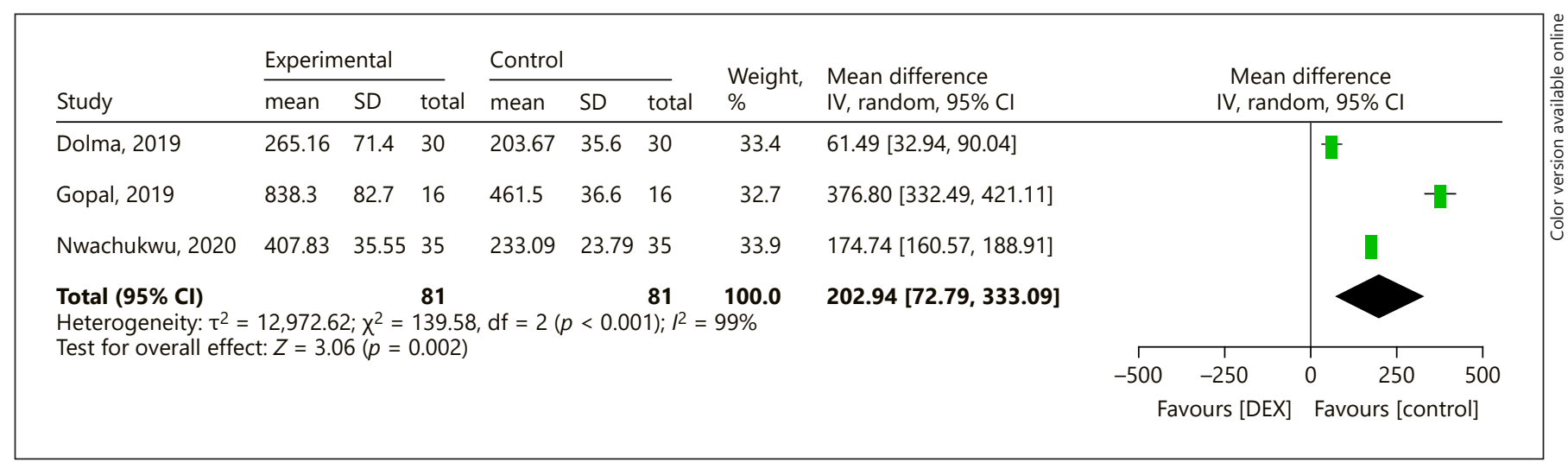

Fig. 3. Effect of DEX compared with control on time to rescue analgesia. DEX, dexmedetomidine.

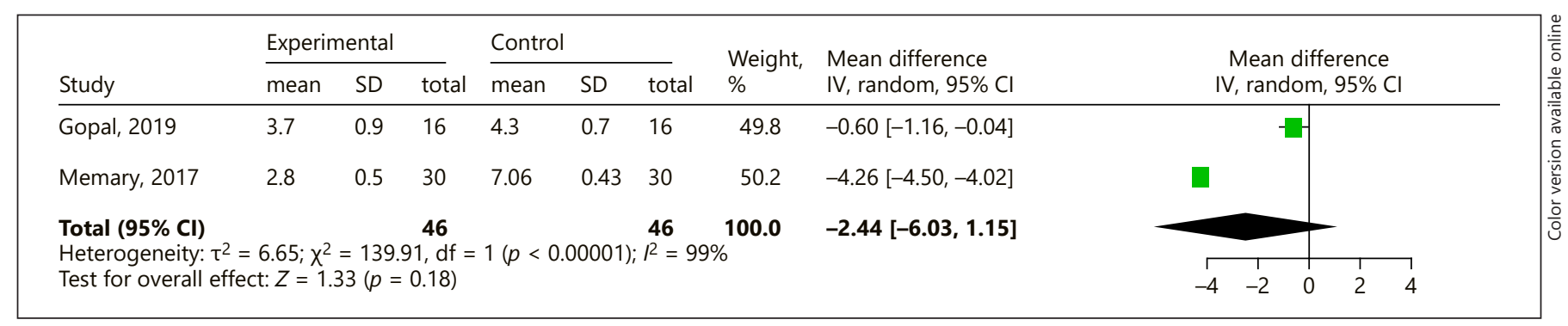

Fig. 4. Effect of DEX compared with control on the visual analog score. DEX, dexmedetomidine.

scores between 2 groups was not statistically significant ( $0.04 ; 95 \%$ CI: -0.07 to $0.15 ; p=0.48)$ with no significant heterogeneity among the studies $\left(I^{2}=18 \%, p=0.27\right)$ (shown in Fig. 5).

\section{Adverse Effects}

Three studies provided the incidence of hypotension in 162 patients. DEX therapy as a local anesthetic adjuvant significantly increased the rate of hypotension compared with control (RR 2.53, 95\% CI: 1.78-3.59, $p=0.02$; Fig. 6). The incidence of postoperative nausea and vomiting was reported in 2 studies. The result showed there was no statistically significant difference in the risks of postoperative nausea and vomiting (RR 2.03, 95\% CI: 0.37 $11.28, p=0.42$; Fig. 7) between anesthetic-with-DEX and the without-DEX therapy. Other adverse events such as bradycardia and shivering are provided in Table 3. No clear evidence of significant difference in the risks of these adverse events was found between 2 groups.

\section{Discussion}

This systematic review and meta-analysis was conducted to estimate the effectiveness and safety of dexmedetomidine for surgery of femur fracture, and eventually 4 RCTs with 222 patients were included. We reported that DEX as an anesthetic adjuvant exhibited a longer duration of rescue analgesia in patients with femur fracture for the first time. However, DEX administration did not provide a sedative sparing effect. Furthermore, the risk of hypotension was markedly increased in the DEX group.

Excruciating pain is one of the most commonly reported discomforts in femur fracture. Even minimal overriding of fracture ends will worsen the pain in these patients. Although many pain-relieving regimens have been employed as regular measures, it is still a challenge to optimize perioperative pain control $[21,22]$. DEX emerges as an attractive alternative to standard anesthetic approaches in recent years. It has specific hypnotic and analgesic properties by the activation of $\alpha 2$-receptors located in the locus coeruleus and spinal dorsal horn [5]. With both 


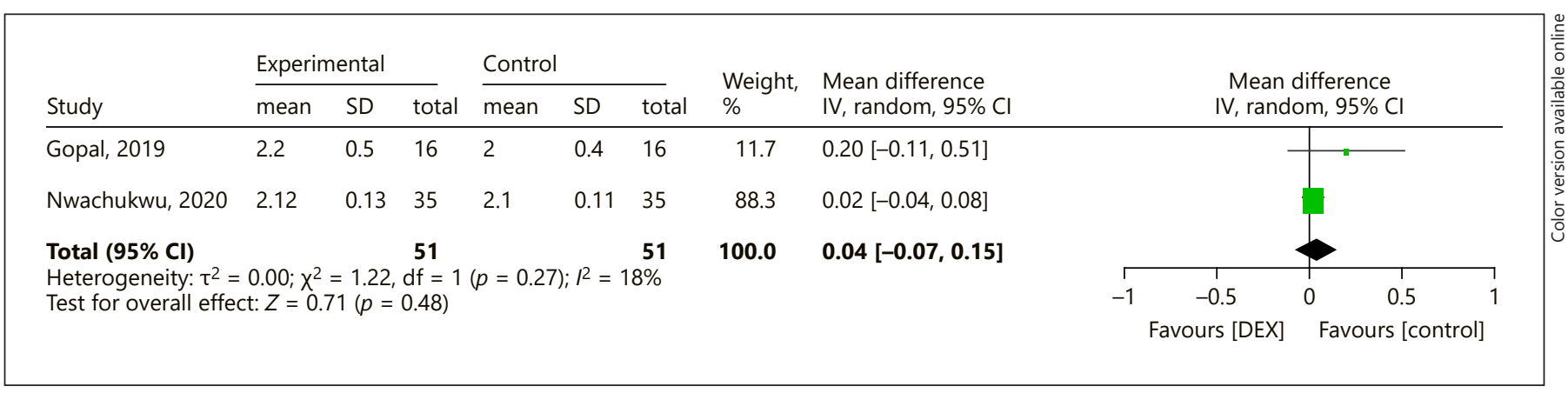

Fig. 5. Effect of DEX compared with control on the sedation score. DEX, dexmedetomidine.

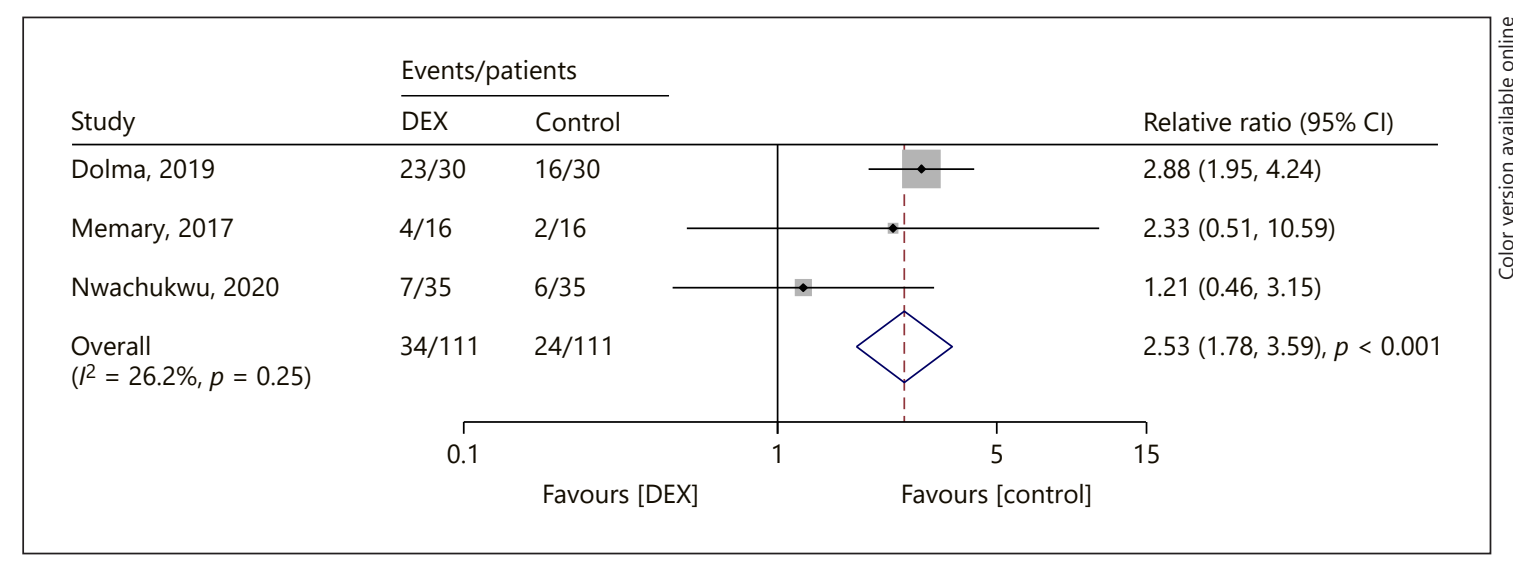

Fig. 6. Effect of DEX compared with control on hypotension. DEX, dexmedetomidine.

\begin{tabular}{|c|c|c|c|c|c|}
\hline \multirow{3}{*}{$\frac{\text { Study }}{\text { Dolma, } 2019}$} & \multicolumn{2}{|c|}{ Events/patients } & & & \multirow[b]{2}{*}{ Relative ratio $(95 \% \mathrm{Cl})$} \\
\hline & DEX & Control & & & \\
\hline & $3 / 30$ & $1 / 30$ & & - & $3.22(0.36,29.13)$ \\
\hline Gopal, 2019 & $1 / 16$ & $0 / 16$ & & $\rightarrow$ & $4.17(0.00,3.54 \mathrm{e}+27)$ \\
\hline Nwachukwu, 2020 & $1 / 35$ & $1 / 35$ & & I & $1.00(0.07,15.34)$ \\
\hline \multirow[t]{3}{*}{$\begin{array}{l}\text { Overall } \\
\left(R^{2}=0.0 \%, p=0.81\right)\end{array}$} & $5 / 111$ & $4 / 111$ & & & $2.03(0.37,11.28), p=0.42$ \\
\hline & & 0.01 & & $10 \quad 30$ & \\
\hline & & & Favours [DEX] & Favours [control] & \\
\hline
\end{tabular}

Fig. 7. Effect of DEX compared with control on nausea and vomiting. DEX, dexmedetomidine.

central and peripheral sympatholytic actions, DEX can be applied as an adjuvant in surgical operation to attenuate perioperative stress and improve the quality of life [12, 23]. In this study, the addition of DEX prolonged the time to rescue analgesic request without affecting the time of sensory block onset, time to achieve maximum sensory block level, and duration of sensory block. As for VAS, there was a general trend of decrease in the DEX group compared to that of without DEX. Gopal and Krishnamurthy [17] found dexmedetomidine infusion signifi- 
Table 3. Adverse events reported in the included RCTs

\begin{tabular}{llllll}
\hline Adverse events & $\begin{array}{l}\text { Studies } \\
\text { reporting, } \\
n\end{array}$ & $\begin{array}{l}\text { DEX } \\
\text { group, } \\
n / N\end{array}$ & $\begin{array}{l}\text { Control } \\
\text { group, } \\
n / N\end{array}$ & RR $(95 \% \mathrm{CI})$ & $\begin{array}{l}p \\
\text { value }\end{array}$ \\
\hline $\begin{array}{l}\text { Bradycardia } \\
\text { Shivering }\end{array}$ & 2 & $8 / 65$ & $5 / 65$ & $1.60(0.49,5.15)$ & 0.43 \\
& 2 & $4 / 65$ & $6 / 65$ & $0.66(0.18,2.47)$ & 0.54 \\
\hline
\end{tabular}

cantly prolonged postoperative analgesia in fracture femur. Nwachukwu et al. [15] showed that a combination of DEX in the management of femoral fractures provided a better anesthetic effect, particularly prolonged duration of postoperative analgesia. A meta-analysis conducted by Yang et al. [13] in patients undergoing total knee or hip arthroplasty revealed dexmedetomidine therapy seemed to be an effective treatment for pain control. These results suggested DEX could serve as an adjuvant to anesthetics to relieve postoperative pain, a practice which ameliorates recovery from anesthesia.

Dexmedetomidine has a sedative effect which is beneficial to minimize intraoperative sympathetic response to a surgical stimulus $[10,24,25]$. Many research studies have agreed the use of DEX as a sedative agent in surgery operation. We observed the sedation score had no statistical significance between the anesthetic-with-DEX group and the without-DEX group in patients with femur fracture surgery. Consistent with our results, Gopal and Krishnamurthy [17] reported DEX infusion did not increase the sedation score in fracture femur. Another study by Nwachukwu et al. [15] also suggested no sedative benefits of dexmedetomidine in femoral fractures. The doses of $0.5 \mu \mathrm{g} / \mathrm{kg}$ and $7.5 \mu \mathrm{g}$ DEX were applied in these studies. Randomized clinical studies of total knee arthroplasty revealed DEX provided a better sedation score in patients with a dose of $1 \mu \mathrm{g} / \mathrm{kg}$ infusion. A plausible reasoning may be due to the low dose used in studies of surgery of femur fracture.

Safety is an important concern with the use of DEX. Hypotension, postoperative nausea, and vomiting are common complications of DEX therapy [26, 27]. Our study found that the incidence of hypotension was increased in the DEX group. DEX as a selective alpha 2-adrenergic agonist which inhibits sympathetic release of catecholamines and prevents protective stress responses to blood pressure decreases, together with which it can increase the vagal activity, leads to the occurrence of hypotension $[7,28]$. Administration of DEX has been shown to result in polyuria and hypotension during the surgical procedure $[29,30]$. The occurrence of hypotension aggravates the risk of limb ischemia and delays the quality of recovery. We need to be cautious about the combination of dexmedetomidine with general or local anesthesia and find ways to reduce the harmful events. There were no significant differences in postoperative nausea and vomiting and other adverse effects between 2 groups. Further research is required to better characterize the underlying mechanisms.

Overall, 4 studies were included and most had a sample size of $<50$ patients; thus, the results might be subject to small study-effect bias. We assessed the evidence as low or moderate certainty for all the comparisons between 2 groups. The main reasons for downgrading the certainty of the evidence are due to inconsistency of findings and imprecision of the effect estimate.

Several limitations should also be considered when explaining our results. First, for most of the comparisons done between 2 groups, the certainty/quality in evidence was mostly evaluated as "moderate" or "low." Second, there was a substantial level of heterogeneity among the results. Different dosage and infusion methods of DEX were compared in these studies, which may affect the credibility of pooling effects. Third, the optimal dosage of DEX in surgery of femur fracture is still a remaining question, which needs to be addressed in future research. Fourth, we restricted the search results to articles published in English language in our inclusion criteria; therefore, the reporting bias could not be ruled out.

\section{Conclusion}

Dexmedetomidine as a local anesthetic adjuvant in femur fracture surgery had a significant longer duration of rescue analgesia. However, the incidence of hypotension was markedly increased in these patients. It was worth noting that the evidence was of low to moderate quality, and more large-sample and high-quality studies are needed to enhance the credibility identified in the present study.

\section{Statement of Ethics}

No approval was required for ethical privacy because the study does not involve direct contact with patients or their personal information.

\section{Conflict of Interest Statement}

The authors declare no conflicts of interest. 


\section{Funding Sources}

No fund was received for this research.

\section{Author Contributions}

Yonghao Yu designed the study; Shan Deng and Yonghao Yu extracted and analyzed data; Shan Deng drafted the manuscript. All authors agree to be accountable for all aspects of the work.

\section{References}

1 Reich MS, Klahs KJ, Fernandez I, Nguyen MP. Alleviation of pain after femur and tibia shaft fractures using nothing stronger than codeine and tramadol. J Orthop Trauma. 2020;34(2):e56-9.

2 Fuzier R, Izard P, Cabos C, Chaminade B, Pouymayou J. Chronic cancer-related pain: continuous perineural infusion of local anesthetics as alternative to systemic analgesic drugs. J Pain Palliat Care Pharmacother. 2016;30:195-200.

3 Al-Melh MA, Andersson L. Comparison of topical anesthetics (EMLA/Oraqix vs. Benzocaine) on pain experienced during palatal needle injection. Oral Surg Oral Med Oral Pathol Oral Radiol Endod. 2007;103:e1620.

4 Bakan M, Umutoglu T, Topuz U, Uysal H, Bayram M, Kadioglu H, et al. Opioid-free total intravenous anesthesia with propofol, dexmedetomidine and lidocaine infusions for laparoscopic cholecystectomy: a prospective, randomized, double-blinded study. Braz J Anesthesiol. 2015;65:191-9.

5 Grewal A. Dexmedetomidine: new avenues. J Anaesthesiol Clin Pharmacol. 2011;27:297302.

6 Dutta S, Lal R, Karol MD, Cohen T, Ebert T. Influence of cardiac output on dexmedetomidine pharmacokinetics. J Pharm Sci. 2000;89: 519-27.

7 Weerink MAS, Struys MMRF, Hannivoort LN, Barends CRM, Absalom AR, Colin P. Clinical pharmacokinetics and pharmacodynamics of dexmedetomidine. Clin Pharmacokinet. 2017;56:893-913.

8 Ebert TJ, Hall JE, Barney JA, Uhrich TD, Colinco MD. The effects of increasing plasma concentrations of dexmedetomidine in humans. Anesthesiology. 2000;93:382-94.

9 MacMillan LB, Hein L, Smith MS, Piascik MT, Limbird LE. Central hypotensive effects of the alpha2a-adrenergic receptor subtype. Science. 1996;273:801-3.

10 Wang L, Zhang T, Huang L, Peng W. Comparison between dexmedetomidine and midazolam for sedation in patients with intubation after oral and maxillofacial surgery. Biomed Res Int. 2020;2020:7082597.
11 Gousheh M, Akhondzadeh R, Rashidi M, Olapour A, Moftakhar F. Comparison of dexmedetomidine and morphine as adjuvants to bupivacaine for epidural anesthesia in leg fracture surgery: a randomized clinical trial. Anesth Pain Med. 2019;9(4):e91480.

12 Ren Y, Shi W, Chen C, Li H, Zheng X, Zheng $\mathrm{X}$, et al. Efficacy of dexmedetomidine as an adjuvant to local wound infiltration anaesthesia in abdominal surgery: a meta-analysis of randomised controlled trials. Int Wound J. 2019;16:1206-13.

13 Yang Q, Ren Y, Feng B, Weng X. Pain relieving effect of dexmedetomidine in patients undergoing total knee or hip arthroplasty: a meta-analysis. Medicine. 2020;99:e18538.

14 Garisto C, Ricci Z, Tofani L, Benegni S, Pezzella C, Cogo P. Use of low-dose dexmedetomidine in combination with opioids and midazolam in pediatric cardiac surgical patients: randomized controlled trial. Minerva Anestesiol. 2018;84:1053-62.

15 Nwachukwu C, Idehen HO, Edomwonyi NP, Umeh B. Postoperative analgesic effect of intrathecal dexmedetomidine on bupivacaine subarachnoid block for open reduction and internal fixation of femoral fractures. Niger J Clin Pract. 2020;23:172-8.

16 Dolma L, Salhotra R, Rautela RS, Banerjee A. Isobaric ropivacaine with or without dexmedetomidine for surgery of neck femur fracture under subarachnoid block. J Anaesthesiol Clin Pharmacol. 2018;34:518-23.

17 Gopal ND, Krishnamurthy D. A clinical comparative study of fascia iliaca compartment block with bupivacaine and bupivacaine with dexmedetomidine for positioning and duration of postoperative analgesia in fracture femur under spinal anesthesia. Anesth Essays Res. 2018 Apr-Jun;12(2):528-34.

18 Higgins JP, Altman DG, Gøtzsche PC, Jüni P, Moher D, Oxman AD, et al. The cochrane collaboration's tool for assessing risk of bias in randomised trials. BMJ. 2011;343:d5928.

19 Guyatt GH, Oxman AD, Vist GE, Kunz R, Falck-Ytter Y, Alonso-Coello P, et al. GRADE: an emerging consensus on rating quality of evidence and strength of recommendations. BMJ. 2008;336:924-6.
20 Memary E, Mirkheshti A, Dabbagh A, Taheri M, Khadempour A, Shirian S. The effect of perineural administration of dexmedetomidine on narcotic consumption and pain intensity in patients undergoing femoral shaft fracture surgery; a randomized single-blind clinical trial. Chonnam Med J. 2017;53:12732.

21 Dell R, Greene D. A proposal for an atypical femur fracture treatment and prevention clinical practice guideline. Osteoporos Int. 2018; 29:1277-83.

22 Oetgen ME, Blatz AM, Matthews A. Impact of clinical practice guideline on the treatment of pediatric femoral fractures in a pediatric hospital. J Bone Joint Surg Am. 2015;97:1641-6.

23 Wang K, Wu M, Xu J, Wu C, Zhang B, Wang $\mathrm{G}$, et al. Effects of dexmedetomidine on perioperative stress, inflammation, and immune function: Systematic review and meta-analysis. Br J Anaesth. 2019;123:777-94.

24 Tsaousi GG, Pourzitaki C, Aloisio S, Bilotta F. Dexmedetomidine as a sedative and analgesic adjuvant in spine surgery: a systematic review and meta-analysis of randomized controlled trials. Eur J Clin Pharmacol. 2018;74:137789.

25 Sun HT, Xu M, Chen GL, He J. [Study of comparing dexmedetomidine and remifentanil for conscious sedation during radiofrequency ablation of hepatocellular carcinoma]. Zhonghua Yi Xue Za Zhi. 2018;98:576-80.

26 PanchgarV, Shetti AN, Sunitha HB, Dhulkhed VK, Nadkarni AV. The effectiveness of intravenous dexmedetomidine on perioperative hemodynamics, analgesic requirement, and side effects profile in patients undergoing laparoscopic surgery under general anesthesia. Anesth Essays Res. 2017;11:72-7.

27 Su F, Hammer GB. Dexmedetomidine: pediatric pharmacology, clinical uses and safety. Expert Opin Drug Saf. 2011;10:55-66.

28 Bloor BC, Ward DS, Belleville JP, Maze M. Effects of intravenous dexmedetomidine in humans. II. Hemodynamic changes. Anesthesiology. 1992;77:1134-42.

29 Xu A, Wan L. Dexmedetomidine-induced polyuric syndrome and hypotension. J Clin Anesth. 2018;44:8-9.

30 Granger S, Ninan D. Intraoperative dexmedetomidine-induced polyuric syndrome. $\mathrm{Cu}$ reus. 2017;9:e1218.
Dexmedetomidine in Surgery of Femur Fracture
Pharmacology 2021;106:453-461

DOI: $10.1159 / 000515788$ 